\title{
Glomus Tumor of Uncertain Malignant Potential: A Rare Case Report
}

\author{
Shailee Mehta, Ritwika Dabral* and Priti Trivedi \\ Department of Pathology, Gujarat Cancer and Research Institute Ahmedabad, India
}

*Correspondence: Ritwika Dabral, Post Graduate Resident, Room 401, Department of Pathology, Gujarat Cancer And Research Institute, Civil Hospital Campus, Asarwa, Ahmedabad-380004, Tel: +919662572985; E-mail: ritwika01@gmail.coms

\begin{abstract}
Glomus tumor is usually a small, benign tumor and typically occurs in the dermis or subcutis or soft tissues of the extremities and rarely in the visceral locations. Its bronchopulmonary origin is exceedingly rare clinical condition. The current case reported a 51-year-old male with dyspnea on exertion and obstructive pneumonia; he had a glomus tumor which has large size, deep location and exhibits an infiltrative margin as well as atypical mitotic figures. These characteristic suggests malignant behavior. Therefore diagnosis of glomus tumor of uncertain malignant potential was favored. Recently, the histopathological diagnostic criteria for malignant glomus tumors were defined in the WHO classification of soft tissue and bone tumors $4^{\text {th }}$ edition. Here we also reviewed the literature on primary bronchopulmonary glomus tumors with special attention to the current concept of malignancy grade estimation.
\end{abstract}

Keywords: Glomus tumor; Lung; Uncertain malignant potential

Received Date: June 19, 2019; Accepted Date: July 02, 2019; Published Date: July 09, 2019

\section{Introduction}

Glomus tumors are relatively rare soft tissue tumors composed of cells that resemble the modified smooth muscle cells of the specialized form of arteriovenous anastomosis "glomus body". The most common site of the tumor is the subungual region; however, they occasionally occur in visceral organs such as airway tracts. Primary glomus tumors of the lung are exceedingly rare. To date, only a few more than 20 cases of tracheobronchial glomus tumor have been reported [1,2] among the total. Here, we report a case of primary pulmonary glomus tumor that arose in right middle lobe of lung protruding as a polypoidal mass in the bronchus with a clinical manifestation of obstructive pneumonia. The malignancy estimation of visceral glomus tumors is a worrisome problem to be addressed because of the rarity of this condition. Recently, the criteria were modified and employed in the WHO classification of soft tissue and bone tumors $4^{\text {th }}$ edition. However, pulmonary glomus tumors diagnosed by the current WHO criteria have been scarcely reported. We describe an additional case of this rare entity.

Citation: Ritwika Dabral, Glomus Tumor of Uncertain Malignant Potential: A Rare Case Report. J Clin Cases Rep 3(1): 14-17. 


\section{Case Presentation}

A 51-year-old man visited the outpatient department of our hospital with chief complaints of dyspnea, fever and was diagnosed with obstructive pneumonia. He had no history of smoking. Although antibiotic treatment was properly initiated, his symptoms persisted. Chest CT examination revealed a $38 \mathrm{~mm} \times 30 \mathrm{~mm}$ soft tissue density lesion in right middle lobe, causing abrupt cut off the branches of the right middle lobe bronchus with resultant distal collapse and consolidation. Physical examination revealed decreased air entry. Routine hematological and chemical laboratory results were normal, except for a slight increase in CRP $(1.0 \mathrm{mg} / \mathrm{dl})$. For confirmation biopsy was done which revealed small round cells arranged in sheets suggestive of glomus tumor. Right pneumenectomy was done for resection of tumor, tumor measures $3.5 \times 2.5 \times 2.0 \mathrm{~cm}^{3}$ well circumscribed, firm and tan in color. Pleura were free from tumor. Microscopically the tumor was composed of monotonus round cells with a centrally placed round nucleus and amphophilic to lightly eosinophilic cytoplasm (Figure 1 and Figure 2) Abundant vascular spaces were observed in the tumor, and some were surrounded by tumor cells. Blood vessels in the tumor are small to medium sized, thin walled resembling capillaries or venules (Figure 3) Occasional mitosis seen (less than 5/50 hpf). Necrosis and lymphovascular emboli are not evident. Subcarinal lymph nodes were not involved by tumor.

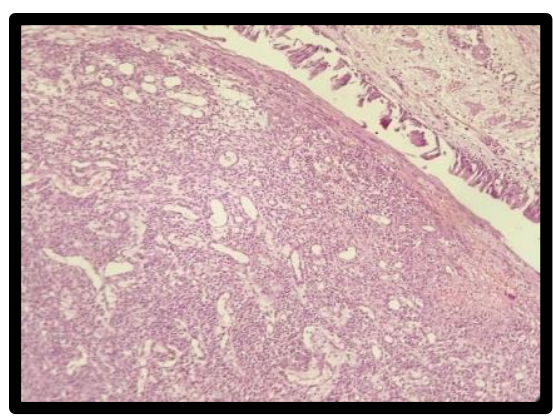

Figure 1: H\&E scanner view.

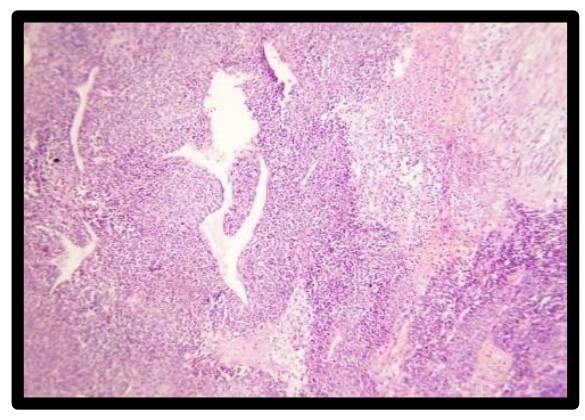

Figure 2: H\&E stain 10X view.

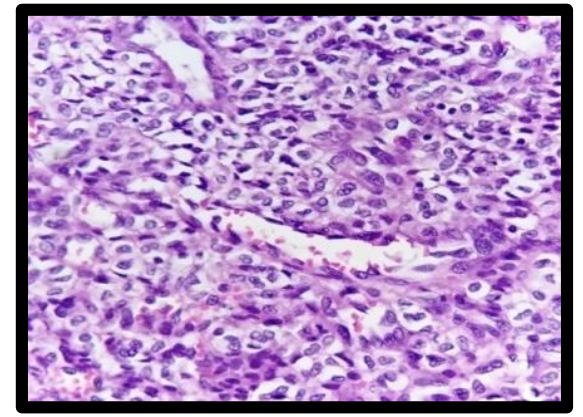

Figure 3: H\&E stain 40X view. 
Immunohistochemically, the tumor cells exhibited cytoplasmic positivity for actin and vimentin (Figure 4 - Figure 6). No immunoreactivity was found for AE1, LCA, CD 20, CD 34, CD 99, FLI1 and synaptophysin.

The patient had an uneventful postoperative course. He had no respiratory complaints and no recurrence of the tumor one year following tumor resection, and then he was lost to follow up.

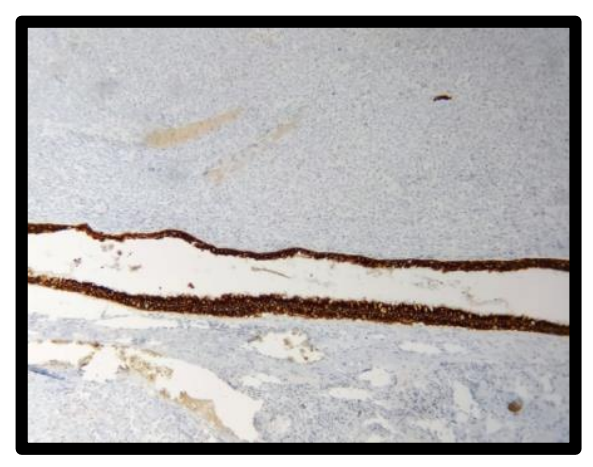

Figure 4: AE 1 negative (10X).

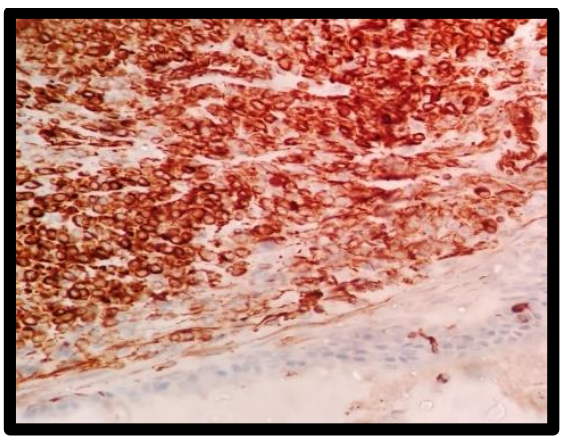

Figure 5: Vimentin positive (40X).

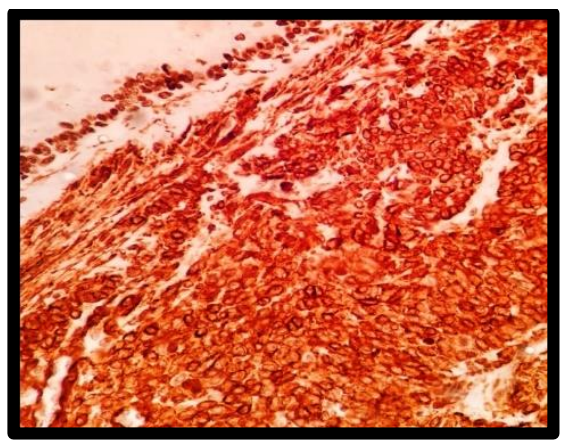

Figure 6: Actin positive (40X view).

\section{Discussion}

The current case reported an extremely rare glomus tumor that was classified as glomus tumor of uncertain malignant potential. The criteria for malignancy of glomus tumors are tumors with a deep location and a size more than $2 \mathrm{~cm}$ or atypical mitotic figures or marked nuclear atypia and 5 or more mitotic figures/50 hpf [3]. In this criterion, the key points indicating malignancy were "large size", "deep location", “atypical mitotic figures", and "nuclear atypia”, but "infiltrative growth pattern" was considered to be unimportant [4]. Current case showed large size, deep location though mitosis was not so 
marked. No distant metastasis was present in our case; therefore, the careful diagnosis of bronchial glomus tumor of uncertain malignant potential was favored.

The diagnosis of glomus tumor of uncertain malignant potential can be elusive. It is necessary to differentiate from hemangiopericytoma and carcinoid tumors especially for glomus tumors in unusual locations. Immunostains for actin and pericellular type IV collagen can confirm the diagnosis of glomus tumor.

Complete resection is the basic procedure for treatment of glomus tumor. In the current case, no metastasis was noted in the 8 lymph nodes below the carina and also no distant metastasis was evident. For one year patient showed no signs of recurrence.

\section{Conclusion}

The current case reported an extremely rare glomus tumor that was classified as glomus tumor of uncertain malignant potential. We further find these neoplasms resemble histologically carcinoid, hemangiopericytoma so careful morphologic observation, histopathological and immunostaining for appropriate markers enable to distinguish between these lesions.

\section{Conflict of Interest}

The authors and planners have disclosed no potential conflict of interest, financial or otherwise.

\section{References}

1. Shang Y, Huang Y, Huang HD, et al. (2010) Removal of glomus tumor in the lower tracheal segment with a flexible bronchoscope: report of two cases. Internal Medicine 49(9): 865-869.

2. Ravenna F, Saturni S, Casalini A, et al. (2011) Bronchial glomus tumor mimicking a COPD exacerbation. Monaldi Archives for Chest Disease 75(3): 194-198.

3. Gill J, Van Vliet C (2010) Infiltrating glomus tumor of uncertain malignant potential arising in the kidney. Human Pathology 41(1): 145-149.

4. Folpe AL, Fanburg-Smith JC, Miettinen M, et al. (2001) Atypical and malignant glomus tumors: analysis of 52 cases, with a proposal for the reclassification of glomus tumors. The American Journal of Surgical Pathology 25(1): 1-12. 